\title{
DISTINGUISHED REPRESENTATIONS FOR UNITARY GROUPS
}

\author{
RAMANUJACHARY KUMANDURI
}

\begin{abstract}
We describe a relative trace formula for the study of distinguished automorphic representations on Unitary Groups with respect to certain Symplectic subgroups. The trace formula will establish that these representations are in correspondence with automorphic representations on $G L(n, K)$ for a quadratic extension $K / k$, which are distinguished with respect to an inner form of $G L(n, k)$. We establish the matching of orbital integrals at finite primes and describe the lifting of spherical representations.
\end{abstract}

\section{Introduction.}

Let $G$ be an algebraic group defined over a number field $k$, and $\pi$ an automorphic representation of $G(\mathbb{A})$. The representation $\pi$ is said to be distinguished with respect to a subgroup $H$ of $G$, if there exists a function $\phi$ in the space of $\pi$, such that the period integral

$$
\Pi_{H}(\phi)=\int_{H(k) \backslash H(\mathbb{A})} \phi(h) d h
$$

is non-vanishing. In many cases, distinguished representations on $G$ with respect to $H$ are in correspondence with representations on another group $G^{\prime}$, distinguished with respect to a subgroup $H^{\prime}$. One approach to the study of these representations is to use Jacquet's relative trace formula, as in [Jac87], [Jac86] or [JL85].

We consider a unitary group of a quadratic extension of number fields: $G=U(n, n)$ with $n=2 r$. $H$ will be a symplectic subgroup in $G$, arising as the fixed points of an involution on $G$. $H$ is the isometry group for a hermitian symmetric form attached to a quaternion algebra $D$ over $k$. The groups and involutions are described in $\S 2$. At almost all places $H$ is the symplectic group $S p(n) \subset G$. We develop a relative trace formula to show that automorphic representations for $G$, distinguished with respect to $H$ are in correspondence with representations on $G^{\prime}=G L(n, K)$ distinguished with respect to an inner form of $H^{\prime}=G L(n, k)$. If $H$ is constructed from a quaternion division algebra $D$, then $H^{\prime}=G L_{r}(D)$. Here $G^{\prime}$ occurs as a Levi 
subgroup of a parabolic subgroup and the local functoriality is parabolic induction.

We expect that if $D$ is a quaternion division algebra then these distinguished representations occur in the cuspidal spectrum. As the local lifting is by parabolic induction from the Siegel parabolic, we expect these representations to be associated to the parabolic in the sense of Piatetski-Shapiro [PS82]. If $D$ is split (i.e., $H \simeq S p(n)$ ), then the distinguished representations occur in the residual spectrum. The residual spectrum for $U(n, n)$ is not completely determined, but it is expected that the part associated to the Siegel parabolic $P$, is given by the residue of a Siegel-type Eisenstein series attached to a cuspidal representation $\sigma$ on $G L(n, K)$. This Eisenstein series can have a pole where the Asai L-function of $\sigma$ has a pole. It is known that the Asai L-function, $L_{\text {Asai }}(s, \sigma)$ has a pole if $\sigma$ is distinguished with respect to $G L(n, k)$. We remark that at Archimedean primes, following the criterion of $[\mathbf{F J} 80]$, there is no discrete series in $L^{2}(G / H)$ if $H \simeq S p(n, \mathbb{R})$, whereas there exist discrete series representations for the symmetric space $G / H$, if $H$ is constructed from the quaternion division algebra.

The related example of $G L(2 n)$ with $S p(n)$-period integrals is studied in [JR92a] and [JR92b]. In this case there are no cuspidal representations which are distinguished. Distinguished representations in the discrete spectrum occur in the residual spectrum [JR92b]. Distinguished representations on $G L(n, K)$ with respect to $G L(n, k)$ are discussed in [Ye89], [JY90] and [Fli91]. These distinguished representations are conjectured to arise as unstable base change lifts from Unitary groups.

We describe the formalism of the relative trace formula for symmetric spaces. Let $\theta: G \rightarrow G$ be an involution defined over $k$. For any $k$-algebra $A, G(A)$ will denote the $A$-points of $G$. Consider the symmetric space

$$
X(\mathbb{A})=\left\{g \in G(\mathbb{A}) \mid \theta(g)=g^{-1}\right\} .
$$

Let $\tau: G \rightarrow G$ be the map $\tau(g)=g^{-1} \theta(g)$ : then the image of $\tau$ is contained in $X$. If $H$ is the group of fixed points of $\theta$, then $\tau(G) \approx H \backslash G$, and the fixed groups of elements of $X$ correspond to inner forms of $H$. We define the action of $G$ on $X$ by $g \cdot x=g^{-1} x \theta(g)$ for $g \in G$ and $x \in X$. This action is compatible with the right action of $G$ on $H \backslash G$.

For the study of distinguished representations, we define a kernel for symmetric spaces as in [JLR93]. Let $f$ be a smooth function on $X(\mathbb{A})$. For $g \in G(\mathbb{A})$, define

$$
K_{f}(g)=\sum_{\xi \in X(k)} f\left(g^{-1} \xi \theta(g)\right)
$$

The projection $K_{f}$ to the space of cuspforms identifies the cuspidal representations with a distinguished vector with respect to an inner form of $H$. 
More precisely, let $\phi$ be an automorphic form on $G$. Consider the integral of $\phi$ against $K_{f}$ and unwind it in the usual way. $H_{\xi}(k)$ denotes the isotropy group of $\xi \in X(k)$.

$$
\begin{aligned}
& \int_{G(k) \backslash G(\mathbb{A})} K_{f}(g) \phi(g) d g \\
& =\int_{G(k) \backslash G(\mathbb{A})} \sum_{\xi \in X(k)} f\left(g^{-1} \xi \theta(g)\right) \phi(g) d g \\
& =\int_{G(k) \backslash G(\mathbb{A})} \sum_{\xi \in X(k) / G(k)} \sum_{\eta \in H_{\xi}(k) \backslash G(k)} f\left(g^{-1} \eta^{-1} \xi \theta(\eta) \theta(g)\right) \phi(g) d g \\
& =\sum_{\xi \in X(k) / G(k)} \int_{H_{\xi}(\mathbb{A}) \backslash G(\mathbb{A})} f\left(g^{-1} \xi \theta(g)\right) \int_{H_{\xi}(k) \backslash H_{\xi}(\mathbb{A})} \phi(h g) d h d g .
\end{aligned}
$$

The inner product $\left\langle K_{f}, \phi\right\rangle$ is nonzero if and only if $\phi$ is distinguished with respect to some inner form of $H$.

Let $B=T U$ be the Levi decomposition of a Borel subgroup, and $\psi$ a character on $U(\mathbb{A})$ trivial on $U(k)$. We consider the component of $K_{f}$ with respect to $\psi$.

$$
\begin{aligned}
& \int_{U(k) \backslash U(\mathbb{A})} K_{f}(u) \psi(u) d u \\
& =\sum_{\xi \in X(k) / U(k)} \int_{U_{\xi}(\mathbb{A}) \backslash U(\mathbb{A})} f\left(u^{-1} \xi \theta(u)\right) \int_{U_{\xi}(k) \backslash U_{\xi}(\mathbb{A})} \psi(u v) d v d u .
\end{aligned}
$$

The inner integral is zero unless $\psi$ is trivial on $U_{\xi}(\mathbb{A})$. We say that the orbit of $\xi$ is relevant for $\psi$ if this condition is satisfied. For a relevant orbit, define

$$
I(\xi, f)=\int_{U_{\xi}(\mathbb{A}) \backslash U(\mathbb{A})} f\left(u^{-1} \xi \theta(u)\right) \psi(u) d u .
$$

Then

$$
\int_{U(k) \backslash U(\mathbb{A})} K_{f}(u) \psi(u) d u=\sum_{\xi} I(\xi, f)
$$

where the sum is over relevant orbits $\xi \in X(k) / U(k)$ for $\psi$.

Let $G$ and $G^{\prime}$ be two groups with involutions $\theta$ and $\theta^{\prime}$. Let $X$ and $X^{\prime}$ denote the corresponding symmetric spaces. Let $\psi$ and $\psi^{\prime}$ be additive characters on the unipotent radicals $U$ and $U^{\prime}$ respectively. Suppose there is a bijection between the relevant orbits, $\xi \mapsto \xi^{\prime}$ and for $f$ on $X(\mathbb{A})$, there is 
$f^{\prime}$ on $X^{\prime}(\mathbb{A})$ such that $I(\xi, f)=I^{\prime}\left(\xi^{\prime}, f^{\prime}\right)$, then one has the relative trace formula

$$
\int_{U(k) \backslash U(\mathbb{A})} K_{f}(u) \psi(u) d u=\int_{U^{\prime}(k) \backslash U^{\prime}(\mathbb{A})} K_{f^{\prime}}^{\prime}\left(u^{\prime}\right) \psi^{\prime}\left(u^{\prime}\right) d u^{\prime} .
$$

If $f=\otimes_{v} f_{v}$, then $I(\xi, f)=\prod_{v} I\left(\xi, f_{v}\right)$ over the places of $k$. For our groups, we show that at finite primes, there is a correspondence between the relevant orbits and given $f_{v}$, a compactly supported smooth function on $X\left(k_{v}\right)$, we construct $f_{v}^{\prime}$ on $X^{\prime}\left(k_{v}\right)$ such that $I_{v}\left(\xi, f_{v}\right)=I_{v}^{\prime}\left(\xi^{\prime}, f_{v}^{\prime}\right)$. This is done in Proposition 5.1.

Our eventual goal is to obtain a formula analogous to (1.7) for the projection $K_{f \text {,cusp }}$ of $K_{f}$ to the space of cuspforms. This would require consideration of the continuous and residual spectrum kernels. We hope to report on this question soon.

The groups under consideration are described in $\S 2$. In $\S 3$, we describe the orbits on symmetric spaces and twisted involutions in Weyl groups. These are used to prove the bijection of relevant orbits in $\S 4$. In $\S 5$, we describe the matching of orbital integrals and the correspondence for spherical representations following [JR92a].

The author would like to thank Prof. Jacquet for his help. Conversations with Prof. Dabrowski were helpful in understanding the results on twisted involutions. Comments of Prof. Rallis regarding this study were helpful.

\section{Description of Unitary and Symplectic Groups.}

Let $K / k$ be a quadratic extension of number fields. For $\alpha \in K$, let $\alpha \mapsto \bar{\alpha}$ be the non-trivial Galois automorphism $K / k$. Choose an element $\delta \in k^{\times}$so that $K=k(\sqrt{\delta})$. For any $k$-algebra $A$, let

$$
G(A)=\left\{g \in G L\left(2 n, K \otimes_{k} A\right): g^{*}\left(\begin{array}{cc}
0_{n} & -1_{n} \\
1_{n} & 0_{n}
\end{array}\right) g=\left(\begin{array}{cc}
0_{n} & -1_{n} \\
1_{n} & 0_{n}
\end{array}\right)\right\} .
$$

The involution $*$ is given by $g^{*}=\bar{g}^{T}$ where the Galois automorphism is extended $A$-linearly to $K \otimes_{k} A$ and applied to all the entries of $g . G(A)$ is the classical unitary group $U(n, n)$.

Let $D$ be a quaternion algebra over $k$ so that $D \otimes_{k} K \simeq M_{2}(K)$. Then there exists an embedding $K \hookrightarrow D$, such that $D=K+K \sigma$, with $\alpha \sigma=\sigma \bar{\alpha}$ and $\sigma^{2}=\gamma \in k^{\times}$. If $\gamma$ is a norm from $K^{\times}$, then $D$ is split. Extend the main involution $\downarrow$ on $D$ to an involution on $M_{n}(D)$ and $M_{2 n}(K)$. We can view $M_{n}(D)^{\times}$as the group of fixed points of an involution on $G L(2 n, K)$. For a suitable choice of the isomorphism $D \otimes_{k} K \simeq M_{2}(K)$, we obtain $M_{n}(D)^{\times}$as 
the fixed point group of the involution $g \mapsto\left(\begin{array}{cc}0_{n} & 1_{n} \\ \gamma_{n} & 0_{n}\end{array}\right) \bar{g}\left(\begin{array}{ll}0_{n} & 1_{n} \\ \gamma_{n} & 0_{n}\end{array}\right)^{-1}$. Then the anti-involution $A \mapsto A^{\star}=A^{T \natural}$ on $M_{r}(D)$ extends to an involution $J g^{T} J^{-1}$ on $G L(2 n, K)$.

Let $H$ be a subgroup of $G L(2 r, D)$ preserving a $\downarrow$-hermitian symmetric $D$-linear form. If $S \in M_{n}(D)$ satisfies $S^{\star}=S$, then

$$
H(A)=\left\{g \in G L_{r}\left(D \otimes_{k} A\right) \mid g^{\star} S g=S\right\}
$$

If $S$ is hyperbolic, then we can view $H$ as a symplectic subgroup of the Unitary group $U(n, n)$. $H(\mathbb{R})$ is the classical group $S p^{\star}(r, r)$, if $D \otimes_{k} \mathbb{R} \simeq \mathbb{H}$. For a given choice of division algebra $D$, corresponding to a choice of $\gamma \in$ $k^{\times} / N_{K / k}\left(K^{\times}\right)$, let $y_{\gamma}=\left(\begin{array}{cc}0_{r} & 1_{r} \\ \gamma 1_{r} & 0_{r}\end{array}\right)$ and $x_{\gamma}=\left(\begin{array}{cc}0 & y \\ -y^{T} & 0\end{array}\right) . H$ is the fixed group of the involution $\theta_{\gamma}: g \mapsto x_{\gamma} g^{T^{-1}} x_{\gamma}^{-1}$ on $G$.

At almost all places $v, \gamma$ is a norm from $K_{v}$, hence the $D \otimes_{k} k_{v} \simeq M_{2}\left(k_{v}\right)$, and $H$ can be considered as the symplectic group $S p(n)$ in $U(n, n)$. In this case we change coordinates and consider the involution $\theta(g)=\bar{g}$. If $K \otimes_{k} k_{v} \simeq k_{v} \times k_{v}$, then $U\left(n, n, K_{v}\right) \simeq G L\left(2 n, k_{v}\right)$. This is the case considered in [JR92a]. If $K \otimes_{k} k_{v} \simeq K_{v}$, a separable quadratic extension of $k_{v}$ and $D$ does not split, then $D$ is the unique quaternion division algebra defined over $k_{v}$ and $H \simeq S p^{\star}\left(r, r, D \otimes k_{v}\right)$.

Let $P=M U$, be the Siegel maximal parabolic in $G$, where the Levi component $M$ consists of matrices

$$
\left(\begin{array}{cc}
a & 0 \\
0 & a^{\star-1}
\end{array}\right)
$$

with $a \in G L(n, K)$. $\theta_{\gamma}$ restricts to an involution $\theta^{\prime}$ on $M$ given by $\theta^{\prime}(a)=$ $y_{\gamma} \bar{a} y_{\gamma}^{-1}$. The fixed group of $\theta_{\gamma}^{\prime}$ is $G L(r, D)$. Using our choices of coordinates it is easy to verify that $P \cap H$ is a maximal proper parabolic in $H$.

On the symmetric space $X^{\prime}(k)=\left\{g \in G L(n, K) \mid \theta^{\prime}(g)=g^{-1}\right\}$, there is only one twisted $G L(n, K)$-orbit. This follows from the vanishing of the Galois cohomology group $H^{1}\left(K / k, G L_{n}\right)$. The corresponding symmetric space for $G, X(k)=\left\{g \in G \mid \theta(g)=g^{-1}\right\}$ also has only one twisted $G$-orbit. This is proved in Corollary 4.2.

\section{Orbits of Unipotents on Symmetric Spaces.}

Our references for the study of orbits of unipotent groups on symmetric spaces are [Spr84] and [HW93]. 
Let $G$ be a reductive group over a local non-Archimedean field $k$, and $\theta$ an involutive automorphism of $G$. Let $H$ be the fixed group of $\theta$,

$$
H=G^{\theta}:=\{g \in G \mid \theta(g)=g\} .
$$

Define the map $\tau: G \rightarrow G$ by $\tau(g)=g^{-1} \theta(g)$. Let $Q$ be the image of $\tau$ and let

$$
X=\left\{g \in G \mid \theta(g)=g^{-1}\right\} .
$$

Then it is easy to verify that $Q \subset X$, and both are closed sub-varieties of $G$. $G$ operates on $Q$ and $X$ by twisted conjugation, $g \cdot x=g^{-1} x \theta(g)$. Then $H \backslash G \approx Q$ as topological $G$-spaces.

Let $B=T U$ be a Borel subgroup, where $U$ is the unipotent radical and $T$ a maximal torus. We study the twisted orbits of $U$ on $X$. Let $A$ be a maximal $\theta$-stable $k$-split torus in $B$. Let $N(A)$ be the normalizer and $Z(A)$ the centralizer of $A$. As usual we define $W=N(A) / Z(A)$. Let $\Phi=\Phi(A, G)$ be the root system with $\Phi^{+}$the positive roots defined by $B$. Let $\Delta$ be the simple system corresponding to $\Phi^{+}$. Let $\Sigma=\left\{s_{\alpha} \mid \alpha \in \Delta\right\}$, and $l$ the length function on the Coxeter system $(W, \Sigma)$.

If $B$ is not $\theta$-stable, then $\theta(B)$ is also a Borel subgroup and there exists $n_{0} \in N(A)$, such that $\theta(B)=n_{0} B n_{0}^{-1}$. Then $\theta\left(\Phi^{+}\right)=w_{0}\left(\Phi^{+}\right)$, if $w_{0}$ is the image of $n_{0}$ in $W$. Then $\theta^{\prime}=\theta w_{0}$ stabilizes $\Phi^{+}$, hence $\Delta$. For computational purposes we can assume $\theta$ stabilizes $\Phi^{+}$.

The following proposition is due to Springer [Spr84, Lemma 4.1] for algebraically closed fields and Helminck and Wang [HW93, Proposition 6.6] for general fields.

Proposition 3.1. If $g \in G$ satisfies $\theta(g)=g^{-1}$, then there exists $x \in U$ such that $x g \theta(x)^{-1} \in N(A)$.

The action of $\theta$ on $\Phi$ induces an automorphism of the Weyl group, given by $\theta(w)=\theta \circ w \circ \theta$. If $s_{\alpha}$ is the reflection associated to $\alpha \in \Phi$, then $\theta\left(s_{\alpha}\right)=s_{\theta \alpha}$. Let

$$
I_{\theta}=\left\{w \in W \mid \theta(w)=w^{-1}\right\}
$$

be the set of twisted involutions in $W$. If $\theta^{\prime}=\theta w$, then $I_{\theta^{\prime}}=I_{\theta} w_{0}$. We note that if $w^{\prime}=w w_{0}$ for $w \in I_{\theta}$, then $w^{\prime} \theta^{\prime}=w \theta$ on $\Phi$. Twisted involutions are decomposed by the following result due to Springer [Spr84, Proposition 3.3]. Part (c) is due to Helminck and Wang, [HW93, Proposition 7.9].

If $I \subset \Delta$, let $W_{I}$ be the group generated by the reflections $s_{\alpha}, \alpha \in I$. Denote the long element of $W_{I}$ by $w_{I}^{0}$.

Proposition 3.2. Let $w \in I_{\theta}$ with $\theta\left(\Phi^{+}\right)=\Phi^{+}$. There exists a $\theta$-stable subset $\Pi$ of $\Delta$ and reflections $s_{1}, s_{2}, \ldots, s_{h} \in \Sigma$ such that:

(a) $\quad w=s_{1} \ldots s_{h} w_{\Pi}^{0} \theta\left(s_{h}\right) \ldots \theta\left(s_{1}\right)$ and $l(w)=l\left(w_{\Pi}^{0}\right)+2 h$. 
(b) $w_{\Pi}^{0} \theta \alpha=-\alpha$ for all $\alpha \in \Phi_{\Pi}$.

(c) If $t_{1}, \ldots t_{m}$ and $\Lambda$ a $\theta$-stable subset also satisfy these conditions for $w$, then $m=h$ and $s_{1} \ldots s_{h} \Pi=t_{1} \ldots t_{h} \Lambda$.

The proposition completely describes the twisted orbits in $I_{\theta}$. Of course, some of these will not occur in $X$.

We remark that in Springer's construction, if $w$ satisfies $s_{\alpha} w \theta\left(s_{\alpha}\right)=w$ for all $s_{\alpha} \in \Sigma$ with $s_{\alpha} w<w$, then the set $\Pi=\{\alpha \in \Delta \mid w \theta \alpha=-\alpha\}$. We prove the following lemma about the decomposition of longest elements in parabolic subgroups of $W$.

Lemma 3.3. Let $J \subset \Delta$ be $\theta$-stable. Then $w_{J}^{0}=w w_{I}^{0} \theta(w)^{-1}$ for some $w \in W$, where $I=\left\{\alpha \in J \mid w_{J}^{0} \theta \alpha=-\alpha\right\}$.

Proof. By compatibility of the action of the different subgroups of $W, w_{I}^{0}$ and $w_{J}^{0}$ have the same action on $\Phi_{I}$.

If $s_{\alpha} w_{J}^{0}<w_{J}^{0}$ and $s_{\alpha} w_{J}^{0} \theta\left(s_{\alpha}\right) \neq w_{J}^{0}$, then $\alpha \in J-I$. Then let $w^{\prime}=$ $s_{\alpha} w_{J}^{0} \theta\left(s_{\alpha}\right)$ for $\alpha \in J-I$. We have $l\left(w^{\prime}\right)=l\left(w_{J}^{0}\right)-2$ and $l\left(s_{\alpha} w^{\prime}\right)>l\left(w^{\prime}\right)$. Therefore the set of $\alpha \in \Delta$ such that $s_{\alpha} w^{\prime}<w^{\prime}$ and $s_{\alpha} w^{\prime} \theta\left(s_{\alpha}\right) \neq w^{\prime}$, is reduced. We repeat this process until we obtain a $w$ such that for $\alpha \in I$, $s_{\alpha} w<w$ and $s_{\alpha} w \theta\left(s_{\alpha}\right)=w$, and for $\alpha \notin I, s_{\alpha} w>w$. Then Springer's construction completes the proof.

Let $U$ be the unipotent radical of $B$, and $\psi$ a character on $U$. We say that an element $n \in N \cap X$ is relevant if $\psi$ is trivial on $U_{n}=\left\{u \in U \mid u^{-1} n \theta(u)=\right.$ $n\}$. A relevant orbit can support a suitable distribution which is left $H_{-}$ invariant and right $(U, \psi)$-equivariant. We describe the group $U_{n}$ and then give a condition for an orbit to be relevant. For $\alpha \in \Phi$, let $\mathfrak{g}_{\alpha}$, be the corresponding root space and $U_{\alpha}$, the additive subgroup of $G$ with Lie algebra $\mathfrak{g}_{\alpha}$. For any subset $C$ of $\Phi^{+}$, define $U_{C}=\prod_{\alpha \in C} U_{\alpha}$. We assume that $U=U_{\Phi^{+}}$.

Definition 3.4. For $w \in I_{\theta}$, define the following subsets of $\Phi$

$$
\begin{aligned}
C(w, \theta) & =\{\alpha \in \Phi \mid \alpha>0, w \theta \alpha>0\} \\
I(w, \theta) & =\left\{\alpha \in \Phi^{+} \mid w \theta \alpha=\alpha\right\} \\
R(w, \theta) & =\left\{\alpha \in \Phi^{+} \mid w \theta \alpha=-\alpha\right\} \\
C^{\prime}(w, \theta) & =C(w)-I(w) .
\end{aligned}
$$

We write $C(w), I(w)$ etc. when there is no ambiguity about the involution $\theta$.

The following proposition is clear from the definition of the isotropy group and of $C(w)$. 
Proposition 3.5. Let $n \in N \cap X$ and $w$ its image in $W$. Then the isotropy group of $n$ for the twisted action of $U$ is $U_{C(w)}^{\xi}$, where $\xi(g)=n \theta(g) n^{-1}$.

Definition 3.6. The support of $\psi$ is defined as a subset $I$ of $\Phi$, such that $\psi\left(U_{\alpha}\right) \equiv 0$ for $\alpha \notin I$. We say that $\psi$ is generic if its support is $\Delta$.

Suppose $\psi$ has support in a subset $J \subset \Delta$. If $\alpha \in J$ and $\alpha \in C(w)$, then $w \theta \alpha=\beta$ must be a simple root and if $w \theta \alpha=\alpha$, then it must be non-compact imaginary with respect to $w$. Otherwise, if $\alpha \in I(w)$, then $U_{\alpha} \subset U(n)^{\xi}$ and $\psi$ does not vanish on $U_{\alpha}$. If $\beta \notin \Delta$, then it is clear that a there is a subgroup of $U_{\alpha} U_{\beta}$ which is $\xi$-stable and $\psi$ doesn't vanish on it. Hence we need $\beta \in \Delta$. We characterize the twisted involutions with this property in the following proposition. See [JR92a, Lemma 1].

Proposition 3.7. Let $w \in I_{\theta}$ and $I=\{\alpha \in \Delta \mid w \theta \alpha \in \Delta\}$. Suppose $I$ is $\theta$-stable and for $\alpha \in \Delta-I, w \theta \alpha<0$, then $\Phi_{I}$ is $w_{\Delta}^{0}$-stable and $w=w_{I}^{0} w_{\Delta}^{0}=$ $w_{\Delta}^{0} w_{I}^{0}$.

Proof. We first show that $w=w_{\Delta}^{0} w_{I}^{0}$. For $\alpha \in I, w \theta \alpha=\beta \in I$, hence $w_{\Delta}^{0} w_{I}^{0} w^{-1} \alpha=w_{\Delta}^{0} w_{I}^{0} \theta \beta>0$ as $I$ is $\theta$-stable. For $\alpha \notin I, w \theta \alpha=\gamma<0$, implies that $w_{\Delta}^{0} w_{I}^{0} w^{-1} \alpha=w_{\Delta}^{0} w_{I}^{0} \theta \gamma>0$ as $w_{I}^{0} \theta \gamma<0$, since $\theta \gamma \notin \Phi_{I}$. This implies that $w=w_{\Delta}^{0} w_{I}^{0}$.

Then $I$ is $\theta$-stable implies that $\theta\left(w_{I}^{0}\right)=w_{I}^{0}$. On the other hand, $w_{\Delta}^{0} w_{I}^{0}$ is a twisted involution, hence $\theta\left(w_{I}^{0}\right)=w_{\Delta}^{0} w_{I}^{0} w_{\Delta}^{0}=w_{J}^{0}$. Hence we conclude that $I=J$. This also shows that $w=w_{\Delta}^{0} w_{I}^{0}$.

The following characterization of $R(w)$ and $C(w)$ will be useful in the sequel. For a proof, see [HW93, Proposition 7.7].

\section{Proposition 3.8.}

(1) If $w=s_{1} s_{2} \ldots s_{h} w_{\Pi}^{0} \theta\left(s_{h}\right) \ldots \theta\left(s_{1}\right)$ is the decomposition of a twisted involution as in Proposition 3.2, then $R(w)=s_{1} s_{2} \ldots s_{h} \Pi$.

(2) If $w \in I_{\theta}$ and $\tilde{w}=s_{\alpha} w \theta\left(s_{\alpha}\right)$, with $l(\tilde{w})>l(w)$, then $I(\tilde{w})=s_{\alpha}(I(w))$ and $C^{\prime}(\tilde{w})=s_{\alpha}\left(C^{\prime}(w)-\{\alpha, w \theta \alpha\}\right)$.

\section{Comparison of Orbits.}

We use the notation of $\S 2$ regarding the groups and involution. Let $x_{\gamma}$ be as defined in $\S 2$, and $\theta$ the corresponding involution. Let $X$ be the associated symmetric space. Let $T$ be the maximal torus consisting of diagonal matrices in $G$ and $S$ the maximal $k$-split torus contained in it. Let $B=T U$ be the standard Borel subgroup consisting of matrices $\left(\begin{array}{ll}a & b \\ 0 & c\end{array}\right)$ with $a$ upper 
triangular. Let $\Phi=\Phi(A, G)$ be the relative root system of $G$ with Weyl group $W=N_{G}(A) / Z_{G}(A)$. As $S$ is $\theta$-stable, $\theta$ operates on $\Phi$ as a linear transformation. There exists $w_{0} \in W$ such that $\theta\left(\Phi^{+}\right)=w_{0}\left(\Phi^{+}\right)$. If $D$ is split, it is easy to verify that there exists $n_{0} \in N \cap X$ such that $n_{0} \mapsto w_{0}$. Hence we can conjugate $\theta$ by $n_{0}$ and assume that $\Phi^{+}$is $\theta$ stable. In fact we can take $\theta$ to be $\theta(g)=\bar{g}$. When $D$ is not split, the same analysis applies for the action on the root system, though all representatives for twisted orbits must now be modified by $w_{0}$, i.e, we consider $\theta^{\prime}=\theta w_{0}$ on $\Phi$, and $I_{\theta}=I_{\theta^{\prime}} w_{0}^{-1}$. If $w^{\prime} \in I_{\theta^{\prime}}$ and $w \in I_{\theta}$, we have $w^{\prime} \theta^{\prime}=w \theta$, hence $C(w, \theta)=C\left(w^{\prime}, \theta^{\prime}\right)$, hence our analysis goes through for this case. Here we can choose $w_{0}=\left(\begin{array}{ll}a & 0 \\ 0 & a\end{array}\right) \in G$ where $a=\left(\begin{array}{ll}0_{r} & 1_{r} \\ 1_{r} & 0_{r}\end{array}\right)$.

Let $\alpha_{1}=t_{1} t_{2}^{-1}, \alpha_{2}=t_{2} t_{3}^{-1}, \ldots \alpha_{n}=t_{n}^{2}$ be the simple roots. $\theta$ action on the roots is trivial. The Lie algebra for $G$ is $\mathfrak{g}=\left\{\left(\begin{array}{ll}a & b \\ c & d\end{array}\right) \in M(2 n, K) \mid a, b, c, d \in M(n, K)\right.$ and $\left.a^{*}=-d, c^{*}=c, b^{*}=b\right\}$.

If $e_{i, j}$ denotes the matrix with a 1 in the $i j$-th position, then the root spaces for $\alpha_{i}$ are $x e_{i, i+1}-\bar{x} e_{i+1+n, i+n}$ for $i<n$ and for $\alpha_{n}$ the root space is $x e_{n, 2 n}$ with $x \in k$. We observe that the root space for $\alpha_{n}$ in $\mathfrak{g}$ is 1-dimensional while the other simple roots have 2-dimensional eigenspaces. The only 1dimensional root spaces are $v_{r}=\alpha_{n}+\alpha_{n-1}+\cdots+\alpha_{n-r}$ for $r=0,1,2, \ldots, n$ and their negatives. If $w \alpha_{n}>0$ for some $w \in W$, then $w \alpha_{n}=v_{r}$ for some $r$. Let $\Pi_{r}=\left\{\alpha_{n}, \alpha_{n-1}, \ldots, \alpha_{n-r}\right\}$.

Proposition 4.1. Let $n \in N \cap X$ and $w$ its image in $W$. Then there exists a $\theta$-stable subset $\Pi \subset \Delta-\left\{\alpha_{n}\right\}$, such that $w=w^{\prime} w_{\Pi}^{0} \theta\left(w^{\prime}\right)^{-1}$ and $w_{\Pi}^{0} \theta \alpha=-\alpha, \forall \alpha \in \Delta$.

Proof. This is essentially due to the fact that $\alpha_{n}$ is a 'non-compact' root relative to $\theta$. To avoid cumbersome notation it is convenient to do the computation using matrices. Given $w$, there exists a $\Pi$ satisfying these conditions from Proposition 3.2. It suffices to show that it is not one of the $\Pi_{r}$ 's. Let $\Pi=\Pi_{r}$ as above. Let $n \in N$ such that $n \rightarrow w_{\Pi}^{0}$ in $W$. Define $n_{\Pi}^{0}=\left(\begin{array}{cccc}1_{n-r} & 0 & 0 & 0 \\ 0 & 0 & 0 & 1_{r} \\ 0 & 0 & 1_{n-r} & 0 \\ 0 & -1_{r} & 0 & 0\end{array}\right)$. Let $n=n_{\Pi}^{0} a$ where $a=\operatorname{diag}\left(a_{1}, a_{2}, \bar{a}_{1}^{-1}, \bar{a}_{2}^{-1}\right)$, where $a_{1}$ and $a_{2}$ are diagonal matrices with $n-r$ elements and $r$ elements respectively. The condition $\theta(n)=n^{-1}$ implies that $\theta(a)=\theta\left(n_{\Pi}^{0}\right)^{-1} a^{-1} n_{\Pi}^{0}$. Evaluating this yields $a_{2}=-a_{2}$, a contradiction. 
Corollary 4.2. There is only one twisted $G$-orbit on $X$.

Proof. Using Proposition 4.1, it suffices to show that $n_{\Pi}^{0}$ is in the twisted $G$-orbit if $n_{\Pi}^{0} \mapsto w_{\Pi}^{0} \in W$, with $\Pi \subset \Delta-\alpha_{n} . n_{\Pi}^{0} \in G L(n, K)$ and satisfies $n_{\Pi}^{0} \bar{n}_{\Pi}^{0}=1$, and by the vanishing of the cohomology $H^{1}\left(K / k, G L_{n}\right)$, there exists $g \in M \subset G$, such that $g \bar{g}^{-1}=n_{\Pi}^{0}$.

If $D$ does not split the proof is similar and there is only one orbit for $G$-action on $X$.

Lemma 4.3. Let $n \in N \cap X$ and $w$ its image in $W$. Then $R(w)$ does not contain any of the $v_{r}$ 's.

Proof. From Proposition 4.1 there exists $\Pi \subset \Delta-\left\{\alpha_{m}\right\}$ such that $w=$ $w^{\prime} w_{\Pi}^{0} \theta\left(w^{\prime}\right)^{-1}$. Then $R(w)=w^{\prime} R\left(w_{\Pi}^{0}\right)=w\left(\Phi_{\Pi}^{+}\right)$. Now $\Pi \subset \Delta-\left\{\alpha_{n}\right\}$, implies that all the root spaces in $\Phi_{\Pi}$ are two dimensional, hence $R(w)$ cannot contain any of the $v_{r}$ 's.

Theorem 4.4. There are no relevant orbits in $N \cap X$ for a generic character $\psi$ on $U$.

Proof. Let $\beta=\alpha_{n}$ be the distinguished root. If $w \theta \beta>0$, it cannot be another simple root, hence $w$ is not relevant. For a relevant orbit we must have $w \theta \beta<0$. Let $I=\{\alpha \in \Delta \mid w \theta \alpha \in \Delta\}$. Now $I \subset \Delta-\{\beta\}$ and $w=w_{I}^{0} w_{\Delta}^{0}$. If $\alpha_{n-1} \notin I$, then $w_{I}^{0} w_{\Delta}^{0} \beta=-\beta$ a contradiction. Suppose there exists an $r$ such that $\alpha_{n-1}, \alpha_{n-2}, \ldots, \alpha_{n-r} \subset I$. Then $v_{r}=\beta+\alpha_{n-1}+\alpha_{n-2}+\cdots+\alpha_{n-r}$, satisfies $w_{I}^{0} w_{\Delta}^{0} v_{r}=-v_{r}$, a contradiction to Lemma 4.3. Hence there are no relevant orbits for a generic character.

The result is equivalent to the fact that there is no generic irreducible admissible representation of $G$ with a linear functional invariant with respect to $H$. See [JR92a, Proposition 3].

We now consider a character $\psi$ supported on $\Delta-\left\{\alpha_{n}\right\}$.

Theorem 4.5. If $w$ is relevant for $\psi$ supported on $\Delta-\left\{\alpha_{n}\right\}$, then $w \in$ $W_{\Delta-\left\{\alpha_{n}\right\}}$.

Proof. If $w$ is relevant we must have $w \theta \alpha_{n}>0$, otherwise we can derive a contradiction as in the proof of Theorem 4.4. We denote $\alpha_{n}$ by $\beta$. Let $w=w^{\prime} w_{\Pi}^{0} \theta\left(w^{\prime}\right)^{-1}$ with $\Pi \subset \Delta-\{\beta\}$.

We will prove that $s_{\beta}$ does not occur in a reduced expression for $w^{\prime}$. Let $\tilde{w}=s_{\alpha} w \theta\left(s_{\alpha}\right)$. If $w \theta \alpha>0$ and not equal to $\alpha$, then $C^{\prime}(\tilde{w})=s_{\alpha}\left(C^{\prime}(w)-\right.$ $\{\alpha, w \theta \alpha\})$. Using this, if $\beta \neq w \theta \beta>0$, then $\beta \notin C^{\prime}\left(s_{\beta} w \theta\left(s_{\beta}\right)\right)$. If $s_{\beta} \gamma=\beta$, then $\gamma=-\beta$ and $-\beta \notin C^{\prime}(w)$. 
On the other hand, if $\tilde{w}=s_{\alpha} w \theta\left(s_{\alpha}\right)$ for $\alpha \neq \beta$, then $\beta \in C^{\prime}(w)$ if and only if $\beta \in C^{\prime}(\tilde{w})$. If $\beta \in C^{\prime}(w)$ and $s_{\alpha} \beta \neq \beta$, then $\alpha=\alpha_{n-1}$. In this case $\alpha_{n-1}, \beta \in C^{\prime}(w)$, implies that $\alpha_{n-1}+\beta \in C^{\prime}(w)$, hence $s_{\alpha_{n-1}}\left(\alpha_{n-1}+\beta\right)=$ $\beta \in C^{\prime}(\tilde{w})$. The converse is similar. Together, these assertions imply that if $w=s_{1} s_{2} \ldots s_{h} w_{\Pi}^{0} \theta\left(s_{h}\right) \ldots \theta\left(s_{1}\right)$ is a reduced expression, then $s_{i} \neq s_{\beta}$ for any $i$. This implies that $w \in W_{\Delta-\left\{\alpha_{n}\right\}}$.

Let $P=M U$ be the parabolic subgroup associated to $I=\Delta-\left\{\alpha_{n}\right\}$. We may choose $M$ so that its Lie algebra is $\Phi_{I}$. Here $M=G L(n, K)$. For $D$ split, $\theta$ is an involution on $M$ given $\theta(g)=\bar{g}$, where $g \in G L(n, K)$. The fixed group of $\theta$ on $M$ is $G L(n, k)$. The restriction of $\psi$ to $M$ gives a generic character on $M$.

If $D$ is not split, then the relevant orbits are of the form $w w_{0}$ with $w$ as in Theorem 4.5. Here $w_{0} \in M$ as described at the beginning of this section.

Theorem 4.6. There is a bijection between relevant orbits for $X$ with respect to the degenerate character $\psi$ and the relevant orbits of $X^{\prime}$ for a generic character $\psi^{\prime}$.

Proof. We have shown that a relevant orbit $n=w a$ for $\psi$ is such that $w \in W \cap$ $M=W_{I}$. We show that $a \in M$. We write $a=\sum n_{\alpha} \alpha^{\vee}$, where $\alpha^{\vee}$ denotes a co-root dual to $\alpha$. Then $\theta(n)=n^{-1}$, implies that $\sum n_{\alpha} w \theta(\alpha)^{\vee}=-\sum n_{\alpha} \alpha^{\vee}$. If $\alpha_{n}^{\vee}$ occurs in the expression for $a$, hence we obtain a contradiction as we have shown that $w \theta\left(\alpha_{n}\right)>0$. This implies that only the roots in $\Delta-\alpha_{n}$ occur in $a$. Hence $a \in M$. Then by the compatibility of $\theta$ action on $X$ and $X^{\prime}$, it is clearly relevant for $X^{\prime}$ with respect to the generic character. Conversely, every relevant orbit for $X^{\prime}$ is clearly relevant for $X$.

\section{Matching of Orbital Integrals.}

Let $G$ be as before with the involution $\theta$. Let $B=T U$ be a Borel subgroup corresponding to a choice of positive roots $\Phi^{+}$with simple system $\Delta$. Let $I$ be a $\theta$-stable subset of $\Delta$. Let $\Phi_{I}$ be the roots generated by $I$ and $P=M V$ be the parabolic subgroup containing $B$ associated to $I$. We choose the Levi component $M$ so that its Lie algebra contains the roots in $\Phi_{I}$ and a Borel subgroup $B_{2}$ of $M$ such that $B=T^{\prime} U^{\prime}$ with positive root system $\Phi_{I}^{+}$. These choices imply that $U=U^{\prime} V$, as a semi-direct product. The involution $\theta$ restricts to an involution on $M$, which we also denote by $\theta$. Let

$$
X^{\prime}=\left\{m \in M \mid \theta(m)=m^{-1}\right\} .
$$

Let $\delta(m)$ denote the module function for the action of $M$ on $V$ for a fixed Haar measure on $V$. For $f$, a smooth function on $X\left(k_{\mathfrak{p}}\right)$, and $\xi \in N \cap M$, 
we define

$$
f^{\prime}\left(m^{-1} \xi \theta(m)\right)=\delta(m) \int_{V_{\xi} \backslash V} f\left(m^{-1} v^{-1} \xi \theta(v) \theta(m)\right) d v .
$$

It is easy to verify that $f^{\prime}$ is a well defined function on $X^{\prime}$. Using this we can show that $f$ and $f^{\prime}$ have matching orbital integrals.

Proposition 5.1. If $f^{\prime}$ is defined as in (5.1) then $f$ and $f^{\prime}$ have matching local orbital integrals, i.e.,

$$
I(\xi, f)=I^{\prime}\left(\xi, f^{\prime}\right)
$$

Proof. This follows from the characterization of the isotropy group in Proposition 3.5. If $\xi$ has image $w \in W$, then $w \theta \alpha>0$ for $\alpha \in \Phi_{I}^{+}$if and only if $w \theta \alpha \in \Phi_{I}^{+}$. Hence we have the factorization $U_{\xi}=U_{\xi}^{\prime} V_{\xi}$, where $U_{\xi}^{\prime}$ and $V_{\xi}$ are the isotropy groups of $\xi$ in $U^{\prime}$ and $V$ respectively. The proposition then follows from

$$
\begin{aligned}
\int_{U_{\xi} \backslash U} f\left(u^{-1} \xi \theta(u)\right) \psi(u) d u & =\int_{U_{\xi}^{\prime} \backslash U^{\prime}} \int_{V_{\xi} \backslash V} f\left(u^{\prime-1} v^{-1} \xi \theta(v) \theta\left(u^{\prime}\right)\right) \psi\left(u^{\prime} v\right) d v d u^{\prime} \\
& =\int_{U_{\xi}^{\prime} \backslash U^{\prime}} f^{\prime}\left(u^{\prime-1} \xi \theta\left(u^{\prime}\right)\right) \psi\left(u^{\prime}\right) d u^{\prime} .
\end{aligned}
$$

Note that the modular function is trivial on $U^{\prime}$ and the restriction of the degenerate character $\psi$ on $U$ is the generic character on $U^{\prime}$.

We now describe the correspondence for spherical representations. Our treatment follows that of [JR92a].

Let $(\sigma, W)$ be an irreducible admissible representation of $G^{\prime}=G L(n, K)$ with a $H^{\prime}=G L(r, D)$ invariant linear form $L$ on $W$. We consider the induced representation $\pi$ on $V=\operatorname{Ind}_{P}^{G} \delta_{P}^{1 / 2} \sigma$ acting by right translations. Our choices of coordinates imply that $P \cap H$ is a maximal proper parabolic in $H$ and $M \cap H=H^{\prime}$. Let $K=G L(n, \mathfrak{O})$ be a maximal compact subgroup of $G$, where $\mathfrak{O}$ is the ring of integers of $K$. Then $K \cap H$ is a maximal compact subgroup of $H$. We define a linear form on $V$ by

$$
T(f)=\int_{K \cap H} L(f(k)) d k
$$

for $f \in V$. Then Iwasawa decomposition in $H$ implies that $T$ is left $H-$ invariant. By definition, if $(\sigma, W)$ is a spherical representation, then $(\pi, V)$ is also spherical. The converse is also true, that every unramified representation of $G$ with a $H$-invariant form is of this form. This is established in [Kum93]. 
Let $H(G, K)$ be the spherical Hecke algebra of $G$. Let $K^{\prime}=G L(n, \mathfrak{O})$ be a maximal compact subgroup of $G^{\prime}$. For $\phi \in H(G, K)$ define $f$ on $X(k)$ as

$$
f\left(g^{-1} \theta(g)\right)=\int_{H} \phi(h g) d h .
$$

For $f^{\prime}$ on $X^{\prime}$ corresponding to $f$ in (5.1) define

$$
\phi^{\prime}\left(g^{\prime}\right)=\int_{K^{\prime}} f^{\prime}\left(g^{\prime-1} k^{-1} \theta(k) \theta\left(g^{\prime}\right)\right) d k
$$

Then one can verify that $\phi^{\prime} \in H\left(G^{\prime}, K^{\prime}\right)$, the spherical Hecke algebra of $G^{\prime}$ and the map $\phi \mapsto \phi^{\prime}$ gives a homomorphism of Hecke algebras.

\section{References}

[FJ80] M. Flensted-Jensen, Discrete series for semisimple symmetric spaces, Annals of Math., 111 (1980), 253-311.

[Fli91] Y. Flicker, On distinguished representations, J. Reine. Angew. Math., 418 (1991), 139-172.

[HW93] A.G. Helminck and S.P. Wang, On rationality properties of involutions on reductive groups, Advances in Mathematics, 99 (1993), 26-96.

[Jac86] H. Jacquet, Sur un résultat de Waldspurger, Annales Scientifiques de l'École Normale Supérieure, 19 (1986), 185-289.

[Jac87] On the non vanishing of some L-functions, Indian Academy of Sciences, Proceedings. Mathematical Sciences, 97 (1987), 117-155.

[JL85] H. Jacquet and K.F. Lai, A relative trace formula, Compositio Math., 54 (1985), 243-310.

[JLR93] H. Jacquet, K.F. Lai and S. Rallis, A trace formula for symmetric spaces, Duke Math. Journal, 70 (1993), 305-372.

[JR92a] H. Jacquet and S. Rallis, Kloosterman integrals for skew symmetric matrices, Pacific J. Math., 154 (1992), 265-283.

[JR92b] _ Symplectic periods, J. Reine Angew. Math., 423 (1992), 175-197.

[JY90] H. Jacquet and Y. Ye, Une remarque sur le changement de base quadratique, Comptus Rendus de l'Academie de Sciences, Paris, 311 (1990), 671-676.

[Kum93] R. Kumanduri, Euler factors of global integrals, Ph.D. Thesis, University of Minnesota, 1993.

[PS82] I.I. Piatetski-Shapiro, Cuspidal representations associated to parabolic subgroups and Ramanujan's conjecture, Number Theory related to Fermat's last theorem, Prog. Math., Birkhauser, Basel-Stuutgart, 1982.

[Spr84] T.A. Springer, Some results on algebraic groups with involutions, Algebraic Groups and Related Topics, Adv. Stud. Pure Math., vol. 6, Academic Press, Orlando, FL, (1984), 525-543. 
[Ye89] Y. Ye, A relative trace formula, J. für Reine Angew. Math., 400 (1989), 57-121.

Received June 27, 1995. The author was partially supported by NSF grant DMS 94-03538.

Departament of Mathematics

COlumbia University

NEW YORK, NY 10027

E-mail address: rama@math.columbia.edu 